\title{
LIFG-based attentional control and the resolution of lexical ambiguities in sentence context
}

\author{
Loan C. Vuong, Randi C. Martin* \\ Department of Psychology, Rice University, TX, United States
}

\section{A R T I C L E I N F O}

\section{Article history:}

Accepted 22 September 2010

Available online 23 October 2010

\section{Keywords:}

LIFG

Attentional control

Lexical ambiguity

Sentence comprehension

\begin{abstract}
A B S T R A C T
The role of attentional control in lexical ambiguity resolution was examined in two patients with damage to the left inferior frontal gyrus (LIFG) and one control patient with non-LIFG damage. Experiment 1 confirmed that the LIFG patients had attentional control deficits compared to normal controls while the non-LIFG patient was relatively unimpaired. Experiment 2 showed that all three patients did as well as normal controls in using biasing sentence context to resolve lexical ambiguities involving balanced ambiguous words, but only the LIFG patients took an abnormally long time on lexical ambiguities that resolved toward a subordinate meaning of biased ambiguous words. Taken together, the results suggest that attentional control plays an important role in the resolution of certain lexical ambiguities - those that induce strong interference from context-inappropriate meanings (i.e., dominant meanings of biased ambiguous words).
\end{abstract}

(c) 2010 Elsevier Inc. All rights reserved.

\section{Introduction}

Language understanding is a cognitive act that involves both automatic and controlled processes (e.g., see Meyer, Wheeldon, and Krott (2007) for discussions). Controlled processes are particularly important when interference arises during interpretation (see Novick, Trueswell, and Thompson-Schill (2005), for a recent review). In this paper, we focus on an internal source of interference - the automatic activation of word meanings - and examine the role of attentional control in the resolution of that interference during sentence comprehension. The issue will be addressed by examining the performance of brain-damaged patients with and without deficits in attentional control.

Certain words have multiple meanings that are unrelated to one another (e.g., bank refers to a financial institution or a river's edge). When two or more meanings have approximately equal association strengths to the ambiguous word form, the word is balanced among alternative meanings. When one meaning is much more strongly associated with the word form than are other meanings, the word is biased with the strongly associated meaning being its dominant meaning and other alternative meanings its subordinate meanings. Due to their association to the word form, unintended alternative meanings may be automatically activated into working memory (WM) as an ambiguous word is processed. This activation of irrelevant meanings is particularly likely when biased

\footnotetext{
* Corresponding author. Address: Psychology Department, MS-25, Rice University, PO Box 1892, Houston, TX 77251, United States. Fax: +1 7133485221.

E-mail address: rmartin@rice.edu (R.C. Martin).
}

ambiguous words are used to refer to a subordinate meaning (e.g., Duffy, Morris, \& Rayner, 1988; Huettig \& Altmann, 2005; Swaab, Brown, \& Hagoort, 2003; Swinney, 1979; see Duffy, Kambe, and Rayner (2001) for review). Significant processing cost, manifested as an increase in reading time on a biased ambiguous word compared to that on an unambiguous control word, has been found for cases in which prior sentence context semantically disambiguates toward the word's subordinate meaning (e.g., Duffy et al., 1988). When prior sentence context disambiguates toward an alternative meaning of balanced ambiguous words, there is no significant processing cost for reading the ambiguous vs. unambiguous control words (e.g., Duffy et al., 1988).

The lack of processing cost for balanced ambiguous words and the significant processing cost for biased ambiguous words have typically been explained by assuming that context and the association strength between the ambiguous word form and an alternative meaning jointly influence the activation level of an alternative meaning (e.g., Duffy et al., 2001). In the case of balanced ambiguous words, each of the equally associated alternative meanings receives an equal amount of activation from the ambiguous word form and prior disambiguating context serves to boost the activation of the intended meaning above that of unintended ones, allowing the intended meaning to be selected immediately as a balanced ambiguous word is processed. In the case of biased ambiguous words, the dominant meaning receives a great amount of activation while subordinate meanings receive little activation from the ambiguous word form. Though prior context adds to the activation of the intended subordinate meaning, the summed activation for the intended subordinate meaning is not sufficient 
for it to be immediately selected as a biased ambiguous word is processed.

Several processes may underlie the processing cost observed for biased ambiguous words presented in prior subordinate biasing sentence context. One source of the cost may be a delay in the resolution toward the context-appropriate subordinate meaning due to competition from the context-inappropriate dominant meaning (e.g., Duffy et al., 2001). This hypothesis presupposes that both the meanings are simultaneously active in WM. Alternatively, the cost may be due to retrieval interference in which the context-inappropriate dominant meaning interferes with the retrieval of the target subordinate meaning (Shivde \& Anderson, 2001). This hypothesis presupposes that the target subordinate meaning has not been sufficiently activated into WM. Perhaps which explanation is correct depends upon the degree of bias, such that competition occurs for biased ambiguous words with less pronounced bias ratios (e.g., word-meaning association strength $=.70 / .30$ for dominant subordinate meaning, respectively) while retrieval interference occurs for biased ambiguous words with more pronounced bias ratios (e.g., .90/.10). Critically, for our purposes, there is a demand for attentional control in both cases, such that comprehenders need to focus attention on contextual factors, which support the intended subordinate meaning, against distraction from the dominant meaning to successfully resolve the competition or retrieval interference from the dominant meaning during lexical ambiguity resolution (Balota, Cortese, \& Wenke, 2001; Novick, Trueswell, \& Thompson-Schill, 2005; Shivde \& Anderson, 2001; Simpson \& Adamopoulos, 2001; Simpson \& Kang, 1994; Thompson-Schill, 2005).

Empirical evidence consistent with the attentional control hypothesis has been obtained in lexical ambiguity resolution studies that involve comprehenders with attentional control deficits (e.g., Balota \& Duchek, 1991; Bedny, Hulbert, \& Thompson-Schill, 2007). Balota and Duchek focused on lexical ambiguity resolution in comprehenders with Dementia of the Alzheimer's Type (DAT) (see Balota and Faust (2001) for a review on attentional control deficits in DAT individuals) and showed that DAT comprehenders had a deficit in lexical ambiguity resolution. The study involved naming of words presented in different contexts (concordant context: music - organ - piano, discordant context: kidney - organ piano, control unrelated context: kidney - ceiling - piano). The target word (piano) was related to the context-appropriate meaning (musical instrument) of the ambiguous word (organ) in the concordant context condition (context word: music), and related to the context-inappropriate meaning (bodily part) of the same ambiguous word (organ) in the discordant context condition (context word: kidney). Similar to normal control comprehenders, DAT comprehenders were significantly faster at target word naming in the concordant vs. unrelated context condition, suggesting that they had no difficulty in activating context-appropriate meanings of ambiguous words. However, unlike normal control comprehenders (who showed no significant difference between the discordant and unrelated condition), DAT comprehenders were still faster at target word naming in the discordant compared to the unrelated context condition, suggesting that they had particular difficulty in resolving interference from context-inappropriate meanings of ambiguous words.

In a different study, Bedny et al. (2007) used a triplet lexical decision task to study lexical ambiguity resolution in a patient with LIFG damage (see Jonides and Nee (2006) for a review on attentional control deficits in LIFG patients). Similar to Balota and Duchek (1991), Bedny et al. (Experiment 2) showed that the LIFG patient had no trouble in activating context-appropriate word meanings; like normal controls, he was significantly faster to make target lexical decision (baby) in the consistent (cow, calf, baby) vs. neutral condition (mirror, cut, baby). However, unlike normal controls, who showed evidence of suppression of contextinappropriate meanings (significantly slower to make target lexical decision in the inconsistent (knee, calf, baby) vs. consistent or neutral condition), the LIFG patient showed no difference between the inconsistent and consistent conditions, suggesting that he activated alternative meanings at a similar level whether they were appropriate or inappropriate to the context.

Together, the word-context studies (Balota \& Duchek, 1991; Bedny et al., 2007) show that comprehenders with attentional control deficits are impaired in lexical ambiguity resolution. The impairment is specific to resolving interference from context-inappropriate meanings rather than to activating alternative meanings of ambiguous words. These findings suggest that attentional control is important for resolving interference from irrelevant word meanings during lexical ambiguity resolution. However, since the studies used only single words as context, it remains to be investigated whether similar evidence for a role of attentional control in lexical ambiguity resolution would obtain if sentence rather word context is used to provide basis for ambiguity resolution.

The goal of our study was to test the attentional control hypothesis of lexical ambiguity resolution using sentence context. Similar to the word-context studies, we tested the hypothesis by focusing on comprehenders with attentional control deficits. Experiment 1 examined attentional control in three patients and showed that two of the patients, who had damage to the LIFG area, had impaired attentional control while the other patient, who did not have LIFG damage, was relatively unimpaired in attentional control compared to normal controls. Experiment 2 examined ability to resolve lexical ambiguities in the patients. We constructed the materials so that prior sentence contexts always disambiguated toward a subordinate meaning of biased ambiguous words or a (slightly less frequent) alternative meaning of balanced ambiguous words. Given the contexts, the (slightly more frequent) alternative meaning of balanced ambiguous words should be minimally interfering while the dominant meaning of biased ambiguous words should likely interfere in lexical ambiguity resolution (e.g., Duffy et al., 1988). The attentional control hypothesis predicts a selective lexical ambiguity resolution deficit in the LIFG patients in the biased ambiguous word condition only.

\section{Patient background}

Two patients - ML and EV, who had lesions that included the LIFG area, and a control patient MB, who had a non-LIFG (parietal) lesion, were tested in both Experiment 1 and 2. Each patient's single word processing and active sentence comprehension were assessed to rule out the possibility that any patient difficulties in sentence processing could be attributed to difficulties with understanding single words or processing simple sentence structures. We used the Peabody Picture Vocabulary Test (choose one of four pictures that matches a spoken word) (PPVT; Dunn \& Dunn, 1981) to assess single word comprehension, the Philadelphia Naming Test (name each single picture with a single word) (PNT: Roach, Schwartz, Martin, Grewal, \& Brecher, 1996) to assess single word production, and a sentence-picture matching task (match each spoken sentence to an appropriate picture with distractor pictures showing a reversal of agent-patient roles or lexical substitutions) to assess comprehension of simple active reversible sentences.

\subsection{LIFG patient $M L$}

ML was a 68-year-old right-handed male, who suffered from a cerebrovascular accident in 1990 that resulted in a lesion comprising the LIFG, frontal areas more superior to the LIFG, and substantial areas of the left parietal lobe (see Fig. 1). His single word 


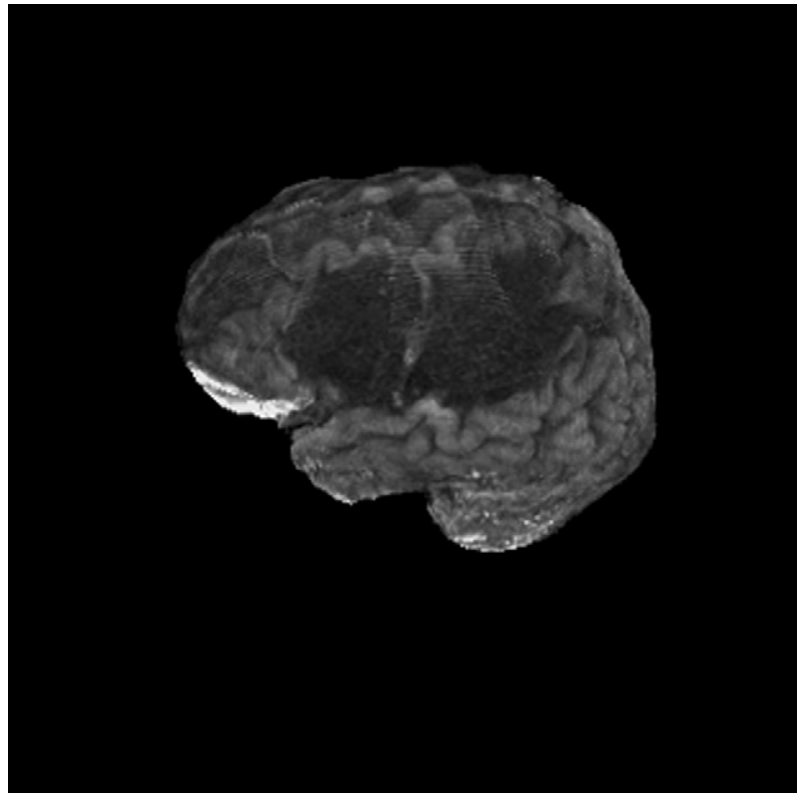

Fig. 1. Structural brain scan of LIFG patient ML.

processing was good (see Martin \& Lesch, 1996); he performed above the mean for normal controls on both the PPVT (107 vs. control mean $=100)$ (Dunn \& Dunn, 1981) and the PNT (98\% correct vs. control mean $=96 \%$ correct $)$ (Roach et al., 1996). He also had good comprehension of simple active reversible sentences, performing at $93 \%$ correct (control mean $=100 \%$ ) on the sentence-picture matching task.

\subsection{LIFG patient EV}

EV was a 53-year-old right-handed female, who suffered from a cerebrovascular accident in 2000 that resulted in a lesion comprising primarily the LIFG at the pars triangularis (BA 45), a small lesion in the left middle frontal gyrus, and an ischemic change in the right parietal lobe (see Fig. 2). Her single word processing

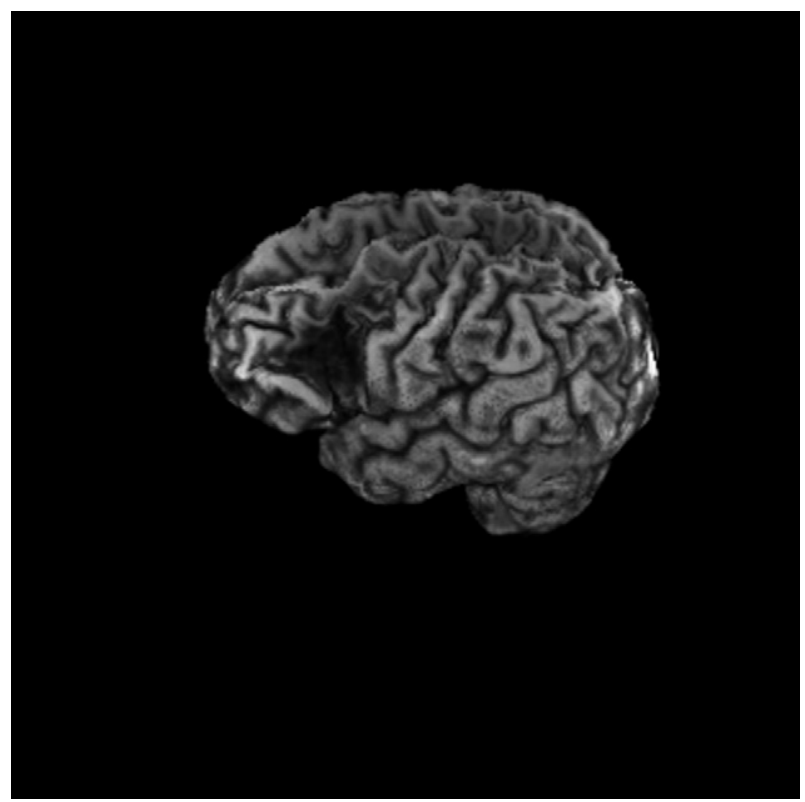

Fig. 2. Structural brain scan of LIFG patient DW. was fair, obtaining a score of 73 on the PPVT (Dunn \& Dunn, 1981) and performing at $85 \%$ correct on the PNT (Roach et al. 1996). Her comprehension of simple active sentences was good, scoring $93 \%$ correct on the sentence-picture matching task.

\subsection{Non-LIFG patient $M B$}

The patient was a 62-year-old right-handed male who suffered from a cerebrovascular accident in 2004 that resulted in a lesion in the left parietal lobe (details obtained from the patient's medical records; brain image unavailable due to possible presence of metal in the patient's body). His single word processing was good, scoring 120 on the PPVT (Dunn \& Dunn, 1981) and performing at $96 \%$ correct on the PNT (Roach et al., 1996). He also had good comprehension of simple active sentences, performing at 93\% correct on the sentence-picture matching task.

Overall, the background results show that the three patients could produce single words and understand single words and simple active reversible sentences adequately.

\section{Experiment 1: attentional control in patients with and without damage to the LIFG}

Three tasks - the Stroop task (Stroop, 1935), the picture-word interference task (Rosinski, Golinkoff, \& Kukish, 1975), and a modified recent-negatives task (Hamilton \& Martin, 2007) - were used to examine attentional control ability in the patients. The critical condition in these tasks induces interference (color word interfering with color ink naming, semantically related word distractors interfering with target picture naming, and semantically and phonological related list items interfering with recognition of probe items, respectively), thus requiring participants to focus attention on task-appropriate representations against distraction from task-inappropriate representations for correct performance. Normal individuals typically show significant interference effects, manifested as a decrease in accuracy and/or an increase in response latency in the critical compared to a control condition, in these tasks (see MacLeod (1991) for a review on Stroop and picture-word interference findings, and Jonides and Nee (2006) for a review on recent-negatives findings). Several LIFG patient cases, including that of ML tested in this study, have recently been documented that showed abnormally large interference effects on one or more attentional control tasks (Barde, Schwartz, \& ThompsonSchill, 2006; Hamilton \& Martin, 2005; Hamilton \& Martin, 2007; Novick, Kan, Trueswell, \& Thompson-Schill, 2009; Thompson-Schill et al., 2002). Together with brain imaging evidence from healthy subjects (e.g., Milham et al., 2003; Jonides et al., 1998), the patient findings argue for a critical role of the LIFG in the implementation of attentional control for interference resolution in WM.

We carried out Experiment 1 for two related purposes. First, we wished to replicate the previous findings on associated relation between LIFG damage and attentional control deficits in a new LIFG patient EV. Second, we sought to confirm that there was indeed a dissociation among our patients on attentional control ability: the non-LIFG patient MB indeed had relatively spared attentional control and the LIFG patients ML and EV indeed had the impairment. A secondary issue was the relation between the patients' attentional control deficits and their STM pattern. Based on the findings from one of the patients reported here (patient $\mathrm{ML}$ ), Hamilton and Martin (2005), Hamilton and Martin (2007) have suggested a link between attentional control deficits and semantic STM deficits, such that reduced semantic STM capacity is caused by impaired attentional control. Thus, the patients were tested on several STM measures as well as the attentional control tasks to address this issue. 


\subsection{Methods}

\subsubsection{Control participants}

Twenty-four healthy control participants were selected from a pool of older adults recruited from the Houston community. Their age ranged from 53 to 79 years old and they had attended at least some college. Not all of the controls were available for testing in all the tasks.

\subsubsection{Stroop task ${ }^{1}$}

Participants saw stimuli presented on the computer screen and named aloud the color of each stimulus as quickly as possible. In the neutral condition (72 trials), they saw asterisks presented in blue, green, orange, purple, red, or yellow. In the incongruent condition (60 trials), they saw color words presented in a different color ink (the word red presented in blue ink). In 12 other trials (congruent fillers), they saw color words presented in the same color ink (the word red presented in red ink). Naming latencies were recorded with a voice key. The order of the trials was prerandomized and fixed for all participants. Interference was defined as the mean difference between incongruent and neutral trials in naming accuracy (neutral minus incongruent) and latency (incongruent minus neutral). The data for patient ML and 10 controls have been reported in Hamilton and Martin (2005).

\subsubsection{Picture-word interference task}

Participants saw pictures on the computer screen that included a word distractor simultaneously presented in each picture. They named the picture and ignored the word. Naming latency was recorded with a voice key. In the related condition (80 trials), the word distractor was semantically related to the picture name (the word banana presented in picture of apple). In the unrelated condition (80 trials), the word distractor was unrelated to the picture name (the word guitar presented in picture of apple). The order of the trials was randomized for each participant. Interference was defined as the mean difference between related and unrelated trials in naming accuracy (unrelated minus related) and latency (related minus unrelated). Fourteen controls performed this task.

\subsubsection{Modified recent-negatives task}

Participants saw lists of three words presented serially on the computer screen, with a probe word presented after each list. The list words were presented for $1000 \mathrm{~ms}$ each, with a betweenword interval of $100 \mathrm{~ms}$. The probe word was presented for $750 \mathrm{~ms}$, with an interval of $1100 \mathrm{~ms}$ between the list and the probe word. Participants indicated as quickly as possible whether the probe appeared in the list (yes) or not (no) by pressing the appropriate key. In the related same list conditions (42 trials per condition), the probe was related to an item presented in the current list, either phonologically (current list: gun, log, hair; probe: pear) or semantically (current list: gun, bar, frog; probe: toad). In the related previous list conditions (42 trials per condition), the probe was related to an item presented immediately before the current list, either phonologically (previous list: gun, log, hair; previous-list probe: tea; current list: hub, book, sky; current-list probe: pear) or semantically (previous list: gun, bar, frog; previous-list probe: tea; current list: hub, book, sky; current-list probe: toad). In the unrelated negative condition (168 trials), the probe was unrelated to the current and immediately previous list items. Besides the 336 negative trials, there were 336 positive filler trials in which the probe word was presented in the current list. The trials were

\footnotetext{
1 The experimental program for this task was provided by Akira Miyake (University of Colorado, Boulder)
}

presented in a fixed prerandomized order and were administered over two testing sessions. Interference was defined as the mean difference between a related negative condition (correct response: "no", interference induced through phonological or semantic relatedness between a list and probe item) and the unrelated negative condition (correct response: "no", minimal interference due to unrelatedness between a current or previous list item and the probe) in response accuracy (unrelated negative minus related negative) and latency (related negative minus unrelated negative). The data for patient ML and 14 controls have been reported in Hamilton and Martin (2007).

\subsubsection{Short-term memory testing}

To further assess the relation between STM deficits and attentional control deficits, we employed several STM tasks, including two semantic STM tasks - three-choice synonymy judgment (decide which two of three words are synonymous) and category probe (decide whether a probe word belongs to the same category as any item in lists with varying number of items) and two phonological STM tasks - forward digit span (hear and repeat, in the original order, lists with varying number of digits) and rhyme probe (decide whether a probe word rhymes with any item in lists with varying number of items). Following Freedman and Martin (2001), we computed a composite STM measure for each STM capacity by first converting each patient's score on each of the STM tasks into a $z$-score (the scores of eight other aphasic patients tested at Rice University and Temple University were included in this analysis), and added together the patient's $z$-scores on the two tasks that measured the same STM capacity to constitute the patient's composite STM score for that STM capacity (phonological vs. semantic).

\subsection{Results}

We first examined attentional control in the control group, by testing for interference effects on each task in the group (significance was determined at the $p<.05$ level, two-tailed). We then tested for attentional control impairments in each patient by comparing the interference effects of each patient to those of the control group. A patient was considered to have impaired attentional control if he or she showed abnormally large interference effects, namely effects that were unlikely to have been drawn from the control distribution, across the three tasks. Significance was determined using the modified $t$-test procedure suggested by Crawford and colleagues (Crawford \& Howell, 1998; see also Crawford et al., 2004). This procedure treats each patient as a sample of $N_{1}=1$, whose score is compared against the scores of the control group under the null hypothesis that the patient's score is an observation from the scores of the control population. Rather than using a $z$-score for this purpose, a $t$-value is computed, which takes into account the size of the control sample. For a patient vs. control comparison that produced a $t$-value exceeding the upper critical $t$-value (at the $p<.05$ level, $d f=N_{2}-1$, one-tailed unless indicated otherwise $)^{2}$, we rejected the null hypothesis and concluded that the patient had shown an abnormally large interference effect. To account for generally longer response times in patients, we

\footnotetext{
2 Given a patient's score $=X_{1}$, the mean of the control group $=X_{2}$, the standard deviation of the control group (estimated population standard deviation) $=S_{2}$, and the control sample size $=N_{2}$, the $t$-value for the patient's score is calculated using the following formula (see Crawford \& Howell, 1998):

$t=\frac{X_{1}-\overline{X_{2}}}{S_{2} \sqrt{\frac{N_{2}+1}{N_{2}}}}$ 
Table 1

STM scores, and mean interference effects (and standard deviations) for normal controls and patients on the attentional control tasks in Experiment 1.

\begin{tabular}{|c|c|c|c|c|}
\hline & \multirow[t]{2}{*}{ Normal controls } & \multirow[t]{2}{*}{ Non-LIFG patient MB } & \multicolumn{2}{|c|}{ LIFG patients } \\
\hline & & & EV & ML \\
\hline \multicolumn{5}{|l|}{ Stroop interference effect } \\
\hline Accuracy & $6.3(6.4)$ & 3.6 & $36.0^{\mathrm{d}}$ & 2 \\
\hline RT & $197(62)$ & 553 & $966^{\mathrm{e}}$ & $979^{\mathrm{e}}$ \\
\hline \multicolumn{5}{|l|}{ Picture-word interference effect } \\
\hline Accuracy & $2.7(2.8)$ & 0 & 3.8 & -1.3 \\
\hline RT & $35(54)$ & -48 & $570^{\mathrm{e}}$ & $623^{e}$ \\
\hline \multicolumn{5}{|l|}{ Recent-negatives interference effect } \\
\hline \multicolumn{5}{|l|}{ Accuracy } \\
\hline Phonologically related same list & $1.9(3.0)$ & -1.2 & 0.6 & $10.7^{\mathrm{d}}$ \\
\hline Phonologically related previous list & $0.3(1.4)$ & -3.6 & 0.6 & $3.6^{\mathrm{d}}$ \\
\hline Semantically related same list & $0.0(1.6)$ & $6.0^{\mathrm{d}}$ & 0.6 & -1.2 \\
\hline Semantically related previous list & $-0.2(1.6)$ & -1.2 & -1.8 & 1.2 \\
\hline \multicolumn{5}{|l|}{ RT } \\
\hline Phonologically related same list & $77(51)$ & -43 & 168 & $387^{\mathrm{e}}$ \\
\hline Phonologically related previous list & $25(70)$ & -34 & 186 & $276^{\mathrm{e}}$ \\
\hline Semantically related same list & $41(38)$ & 21 & $183^{\mathrm{e}}$ & $356^{\mathrm{e}}$ \\
\hline Semantically related previous list & $5(60)$ & -21 & 103 & $494^{\mathrm{e}}$ \\
\hline Phonological STM composite & & 0.11 & 0.28 & -1.74 \\
\hline Forward digit span & $6.0^{c}$ & 3.5 & 7 & 3.5 \\
\hline Rhyme probe & $7.5^{\mathrm{b}}$ & 5 & 3.3 & 1.8 \\
\hline Semantic STM composite & & 0.32 & -0.61 & -0.61 \\
\hline Synonymy judgment & a & $88 \%$ & $83 \%$ & $83 \%$ \\
\hline Category probe & $6.1^{\mathrm{b}}$ & 2.5 & 1.8 & \\
\hline \multicolumn{5}{|c|}{ a Control results were not available. } \\
\hline \multicolumn{5}{|c|}{ b Results reported in Freedman and Martin (2001). } \\
\hline \multicolumn{5}{|c|}{${ }^{\mathrm{c}}$ Highest list length tested on the controls, results reported in Martin, Lesch, and Bartha (1999). } \\
\hline \multicolumn{5}{|c|}{ d Abnormally large interference effects on accuracy ( $p$ against controls <.05). } \\
\hline e Abnormally large interference effects & & & & \\
\hline
\end{tabular}

compared the RT interference effects of each patient against the control means on both untransformed and transformed data. For the untransformed RT analyses, data points that were $2.5 \mathrm{SDs}$ beyond the mean of each condition in each subject were replaced with the 2.5SD cutoff value. For the transformed RT analyses, all data (untrimmed) were included and log-transformed prior to analysis (see Verhaeghen \& De Meersman, 1998). A summary of the results is provided in Table 1.

\subsubsection{Stroop task}

As reported in Hamilton and Martin (2005), the control group showed a significant interference effect of $6.3 \%(S D=6.4)$ on naming accuracy $(t(9)=3.09, p=.01)$ and a significant interference effect of $197 \mathrm{~ms}(S D=62)$ on naming latency $(t(9)=-10.0$, $p<.0001)$. For naming accuracy, non-LIFG patient MB showed a normal 3.6\% effect ( $t$ against controls $<1$ ), LIFG patient EV an abnormally large $36 \%$ effect $(t(9)=4.40, p<.001)$, and LIFG patient ML a normal $2 \%$ effect $(t<1)$. For naming latency, non-LIFG patient MB showed a $553 \mathrm{~ms}$ effect (which was abnormally large in the untransformed data: $t(9)=5.47, p<.001$, but not after log-transformation: $t(9)=1.41, p=.10)$. In contrast, LIFG patient EV showed a $966 \mathrm{~ms}$ effect and LIFG patient ML a 979 ms effect, both of which were abnormally large in both untransformed $(t(9)=11.82$ and 12.03, respectively, both $p s<.001$ ) and log-transformed data $(t(9)=1.89$ and 1.86 , respectively, both $p s<.05)$.

\subsubsection{Picture-word interference task}

The control group showed a significant interference effect of $2.7 \%(S D=2.8)$ on naming accuracy $(t(13)=3.61, p=.003)$ and a significant interference effect of $35 \mathrm{~ms}(S D=54)$ on naming latency $(t(13)=2.43, p=.03)$. Compared to controls, non-LIFG patient MB showed a normal 0\% effect, LIFG patient EV a normal 3.8\% effect, and LIFG patient ML a normal reverse $-1.3 \%$ effect on naming accuracy (all three ts against controls <1). For naming latency, non-LIFG patient $\mathrm{MB}$ showed a normal reverse $-48 \mathrm{~ms}$ effect (not significantly different from the control mean: $t(13)=-1.50$ and -.90 for untransformed and log-transformed data, respectively, $p s>.15$, two-tailed), while LIFG patient EV showed a 570 ms effect and LIFG patient ML a 623 ms effect, both of which were abnormally large in both untransformed $(t(13)=9.66$ and 10.61 , respectively, $p s<.001)$ and log-transformed data $(t(13)=$ 3.16 and 3.47, respectively, ps $<.004$ ).

\subsubsection{Modified recent-negatives task}

The control group showed a significant interference effect of $1.9 \%(S D=3.0)$ on response accuracy for phonologically related same list trials only $(t(13)=2.32, p=.04)$; they showed non-significant interference effects in the other three related conditions: .3\% effect $(S D=1.4)$ for phonologically related previous list, $0 \%$ effect $(S D=1.6)$ for semantically related same list, and reverse $-.2 \%$ effect $(S D=1.6)$ for semantically related previous list trials (all three ts against controls $<1$ ). For accuracy, non-LIFG patient MB showed small, normal reverse effects in all the conditions, except for the semantically related same list condition in which he showed a $6.0 \%$ interference effect that was abnormally large relative to controls $(t(13)=3.71, p=.001)$. LIFG patient EV did not show abnormally large interference effects on accuracy in any condition (all ts $<1$ ). LIFG patient ML showed abnormally large interference effects in the two phonologically related conditions only $(10.7 \%$ effect for phonologically related same list and 3.6\% effect for phonologically related previous list trials, $t(13)=2.83$ and 2.18 , respectively, $p s<.03$ ).

For response latency, the controls showed significant interference effects in the two related same list conditions (phonologically related same list: $77 \mathrm{~ms}$ effect, $S D=51, t(13)=5.57, p<.001$, and semantically related same list: $41 \mathrm{~ms}$ effect, $S D=38, t(13)=4.00$, 
$p=.002)$; they showed non-significant interference effects of $25 \mathrm{~ms}(S D=70)$ for phonological related previous list and $5 \mathrm{~ms}$ effect $(S D=60)$ for semantically related previous list trials ( $t$ < < 1.4). Non-LIFG patient MB did not show abnormally large interference effects in any condition in either untransformed or log-transformed data (all ts against controls <1). LIFG patient EV showed an abnormally large interference effect of $183 \mathrm{~ms}$ in the semantically related same list condition in both untransformed and log-transformed data $(t(13)=3.62$ and $2.20, p=.002$ and .02 respectively) and an interference effect of $186 \mathrm{~ms}$ in the phonologically related previous list condition that was abnormally large in the untransformed data only $(t(13)=2.23, p=.02$; after logtransformation: $t(13)=1.14, p=.14)$. LIFG patient ML showed abnormally large interference effects in all four conditions in both untransformed and log-transformed data (all ps <.03).

\subsubsection{STM}

LIFG patient ML obtained negative composite scores for both the phonological and semantic STM components ( -1.74 for phonological and -.61 for semantic), suggesting that he had worse phonological and semantic retention relative to the other patients in the sample ${ }^{3}$. LIFG patient EV obtained a positive composite score on the phonological STM component (.28) and a negative composite score on the semantic STM component (-.61), suggesting better phonological retention and worse semantic retention than the other patients in the sample. Non-LIFG patient MB obtained positive composite scores for both the phonological (.11) and semantic (.32) components, suggesting better retention on both the STM components compared to the other patients.

\subsection{Discussion of Experiment 1}

In line with previous findings (Barde et al., 2006; Hamilton \& Martin, 2005; Hamilton \& Martin, 2007; Thompson-Schill et al., 2002), the LIFG patient EV (and ML) consistently showed abnormally large interference effects across all three attentional control tasks while the non-LIFG patient MB did not. These results added further evidence to the hypothesis that the LIFG serves to resolve interference in WM (Jonides \& Nee, 2006).

With regard to the STM results, the LIFG patients ML and EV both had impaired semantic STM ability and both showed exaggerated interference effects in the attentional control tasks. Thus, the results are consistent with the Hamilton and Martin's view that attentional control and semantic STM deficits may be causally linked (but see Barde et al., 2006 and Barde, Schwartz, Chrysikou, \& Thompson-Schill, 2010). However, it should be noted that the non-LIFG patient MB's semantic composite was only slightly higher than that of ML or EV but he showed substantially reduced interference effects relative to these patients. Data from further patients would be needed to determine the extent to which semantic STM deficits and deficits in the resolution of interference in WM are dissociable.

\section{Experiment 2: lexical ambiguity resolution in patients with and without LIFG damage}

Experiment 1 showed that the LIFG patients ML and EV had attentional control deficits while the non-LIFG patient MB had relatively normal attentional control. Experiment 2 was carried out to examine their ability to resolve lexical ambiguities during sentence

\footnotetext{
${ }^{3}$ Compared to previous reports, ML's phonological STM capacity has worsened substantially (current rhyme probe span $=1.8$ items vs. 3 items as reported in Freedman \& Martin, 2001). His semantic STM impairment remained severe (current category probe span $=1.5$ items vs. 1.8 items as reported in Freedman \& Martin, 2001).
}

processing. The patients and a group of normal older controls read one sentence at a time at their own pace and named a word after finishing reading each sentence. The experimental materials consisted of short active sentences that ended with an ambiguous word or an unambiguous control word. Half of the ambiguous words were biased toward a particular alternative meaning while the other half were balanced between two alternative meanings. Prior sentence context always semantically disambiguated toward the less frequent meaning of the ambiguous words, which was the subordinate meaning in the case of biased ambiguous words (high interference from the dominant meaning) and the slightly less frequent alternative meaning in the case of balanced ambiguous words (low interference from the slightly more frequent alternative meaning). Each experimental sentence was followed by a word to be named, which was always related to the context-inappropriate meaning of the ambiguous word (see example).

$\begin{array}{ll}\text { Biased ambiguous words (high interference) } \\ \text { He drank the port } & \text { DOCK (ambiguous) } \\ \text { He drank the wine } & \text { DOCK (unambiguous) } \\ \text { Balanced ambiguous words (low interference) } \\ \begin{array}{ll}\text { She mixed the punch } & \text { BLOW (ambiguous) } \\ \text { She mixed the drink } & \text { BLOW (unambiguous) }\end{array}\end{array}$

The dependent measures were sentence reading time and word naming latency. The difference in sentence reading time in an ambiguous vs. unambiguous condition (ambiguity effect $=$ ambiguous minus unambiguous) provided a measure of word meaning interference, and critically, resolution of that interference during sentence comprehension. The difference in word naming latency in an ambiguous vs. unambiguous condition (ambiguity effect $=$ ambiguous minus unambiguous) provided a measure of residual activation of context-inappropriate word meaning following lexical ambiguity resolution, and therefore, success at lexical ambiguity resolution during sentence comprehension. To determine whether the patients were impaired in lexical ambiguity resolution, we compared the ambiguity effects of each patient against those of normal age-matched (older) controls on each of the dependent measures.

\subsection{Predictions for the control group}

As our sentence context was strongly biased toward the intended meaning and we used a self-paced sentence reading task that allowed participants to spend as much time as needed in contextual processing, we might expect the older controls to show a pattern of effects on sentence reading time that is similar to that found in younger comprehenders (see Balota et al. (2001) and Dagerman, MacDonald, and Harm (2006) for discussions). Specifically, we might expect that prior disambiguating sentence context would be effective in constraining lexical access to the contextappropriate meaning in the balanced ambiguous word condition but not in the biased ambiguous word condition, resulting in no significant ambiguity effect on sentence reading time (similarly fast reading of ambiguous vs. unambiguous sentences) in the balanced ambiguous word condition and a significant effect of ambiguity (slower reading of ambiguous vs. unambiguous sentences) in the biased ambiguous word condition for the controls.

Prior studies on aging that used the word naming task to measure activation of context-inappropriate meanings (e.g., Hopkins, Kellas, \& Paul, 1995; Paul (1996); see Burke (1997) for a review) have shown no significant priming of context-inappropriate meanings in older adults following ambiguity resolution, even if the inappropriate meanings were the dominant meanings of biased 
ambiguous words. Consistent with these findings, we might expect the older controls to show no significant priming of context-inappropriate meanings (i.e., no significant ambiguity effect on word naming latency) for both the balanced and biased ambiguous word condition.

\subsection{Predictions for the patients}

According to the attentional control hypothesis, attentional control plays an important role in resolving interference from context-inappropriate word meanings during lexical ambiguity resolution. Given that the context-inappropriate dominant meaning of biased ambiguous words interferes in lexical ambiguity resolution while the context-inappropriate alternative meaning of balanced ambiguous words does not, the attentional control hypothesis predicts that the LIFG-based attentional control patients would show impaired lexical ambiguity resolution in the biased ambiguous word condition only. The patients may, due to their impaired attentional control, become less efficient at resolving against the dominant meaning of biased ambiguous words and/or, more severely, fail to resolve the ambiguity eventually. A decrease in efficiency may cause them to take an abnormally long time in resolving the ambiguity while resolution failure may lead to sustained activation of the dominant meaning following (failed) ambiguity resolution.

We thus predicted that (i) LIFG patients ML and EV would show at least an abnormally large ambiguity effect on sentence reading in the biased ambiguous word condition; they may additionally show an abnormally large ambiguity effect on word naming in the biased ambiguous word condition, (ii) LIFG patients ML and EV would show normal ambiguity effects in the balanced ambiguous word condition, and (iii) non-LIFG patient MB, thanks to his relatively normal attentional control ability, would show normal ambiguity effects in both the biased and balanced ambiguous word condition. Alternatively, if attentional control plays no role in lexical ambiguity resolution under any interference condition, all three patients should show normal ambiguity effects in both the ambiguous word type conditions.

\subsection{Methods}

\subsubsection{Participants}

Patients ML, EV, and MB were tested along with 20 normal controls (mean age $=63.6, S D=5.9$ ). The controls were divided into two non-overlapping subgroups to better match with the age of the patients (the control group for ML: $n=10$, mean age $=68.6$, the control group for EV and MB: $n=10$, mean age =58.5). All of the participants were tested over two sessions that were approximately 1 week apart.

\subsubsection{Materials}

The materials consisted of 28 short (4-6 words long) experimental sentence contexts, which were manipulated for two factors, ambiguity and type of ambiguous words (henceforth, word type). Ambiguity was a within-item factor, as the same sentence context was constructed to end with an ambiguous word or an unambiguous control word. Word type was a between-item factor, as an ambiguous word was either biased or balanced. Both ambiguity and word type were within-participant factors, as each participant was tested in all four conditions (biased ambiguous, biased unambiguous, balanced ambiguous, balanced unambiguous).

Word type was verified through a separate free word association norming study with 76 undergraduates at Rice University. The mean frequency of the context-appropriate vs. context-inappropriate meaning was, respectively, $11 \%$ vs. $78 \%$ for biased ambiguous words, and $33 \%$ vs. $42 \%$ for balanced ambiguous words.
Contextual bias towards the appropriate meaning was verified through a separate plausibility rating task performed by 20 Rice University undergraduates ( 7 highly plausible -1 highly implausible). The rating results confirmed that degree of contextual bias was similar across the word types (mean plausibility rating $=6.64$ and 6.72 for biased and balanced ambiguous words, respectively) $(F<1)$. The character length of the sentence final word was also similar across the ambiguous vs. unambiguous condition for biased ambiguous words (mean length $=4.50$ and 4.50 , respectively, $F<1$ ) and balanced ambiguous words (mean length $=4.57$ and 4.71 , respectively, $F<1$ ). In addition, the log frequency of the sentence final word was similar across the ambiguous vs. unambiguous condition for biased ambiguous words (mean $\log$ frequency $=2.79$ and 3.39 , respectively, $F<1.5$ ) and balanced ambiguous words (mean $\log$ frequency $=3.37$ and 3.43 , respectively, $F<1$ ). Finally, across the word types, the words to be named were similar in length (mean character length $=4.43$ and 4.21 for biased and balanced ambiguous words, respectively) and log frequency (mean log frequency $=3.71$ and 3.70 for biased and balanced ambiguous words, respectively) $(F s<1)$.

A total of 14 sentence contexts were created for each set of word types. Two lists of materials were constructed, such that each list contained an equal number of items from each of the four ambiguity $\times$ word type conditions. No items were repeated within a list but across the two lists each item was presented twice, once in each ambiguity version. In addition to the experimental items, each list contained 28 filler items. Half of the filler sentences ended with an ambiguous word (e.g., He repainted the cabinet). Each filler sentence was also followed by a word to be named, which was not related to the sentence meaning (e.g., VOICE). Each participant was tested on both lists and list order was counterbalanced for the control group.

\subsubsection{Procedure}

Each trial started with a row of asterisks presented at the center of a computer screen. When ready, participants pressed a key to read a sentence. Once they had finished reading the sentence, they pressed a key to move onto the next event in the trial. Sentence reading time was recorded as the duration of time between the two key presses. For the experimental trials and half of the filler trials, a word to be named appeared next. Participants were instructed to name the word as quickly and accurately as possible into a microphone. Word naming latency was recorded as the duration of time between the presentation of the word and the triggering of the voice key. To make sure that participants carefully read the sentences, in the other half of the filler trials $(1 / 4$ th of all trials), immediately after finishing reading the sentence context and pressing the key to advance, participants received a prompt asking them to create a new sentence that was related to the reading context. For these trials, the word naming event occurred right after the sentence creation event. Neither the controls nor the patients had difficulty performing the filler task.

\subsection{Results}

We first examined lexical ambiguity resolution in the control group $(n=20)$, by testing for ambiguity effects across word types and dependent measures in the group (planned comparisons were done between ambiguous vs. unambiguous conditions, significance was determined at the $p<.05$ level, two-tailed). We then tested each patient's ambiguity effects against those of the appropriate control group. A patient was considered to have impaired resolution for a certain type of lexical ambiguity if he or she showed abnormally large ambiguity effect(s) for that word type condition. Similar to Experiment 1, an abnormally large ambiguity effect is one having a probability of $p<.05$, one-tailed, of being 
Table 2

Mean RTs per condition and mean ambiguity effects (and 95\% confidence interval) for the control group ( $n=20)$ on each dependent measure of the lexical ambiguity resolution task in Experiment 2.

\begin{tabular}{lllll}
\hline & Word type & Ambiguous & Unambiguous & Ambiguity effect \\
\hline \multirow{2}{*}{ Sentence reading } & Biased & 2359 & 2211 & $148(39-257)^{* *}$ \\
& Balanced & 2149 & 2161 & $-12(-84$ to 61$)$ \\
\multirow{2}{*}{ Word naming } & Biased & 835 & 843 & $-7(-38$ to 23) \\
& Balanced & 834 & 840 & $-6(-39$ to 27) \\
\hline
\end{tabular}

Ambiguity effect $=$ ambiguous minus unambiguous

$p<.05$ by subjects and items.

drawn from the control distribution under Crawford and Howell's (1998) modified $t$-test procedure. Also similar to Experiment 1, we accounted for general slowing in some patients (which was noteworthy particularly for ML) by log-transforming the data of all participants and performing the patient vs. control comparisons on those data (see Verhaeghen \& De Meersman, 1998). For the untransformed data, data points that were 2.5 SDs beyond the mean of each condition by each subject were replaced with the $2.5 S D$ cutoff value (less than $2 \%$ of the data were affected). For the log-transformed data, all untrimmed data were included. Results for the control group are summarized in Table 2. Results for the patients are summarized in Table 3 .

\subsubsection{Control group}

As expected, normal controls showed a significant ambiguity effect of $148 \mathrm{~ms}$ on sentence reading in the biased ambiguous word condition (ambiguous: $2359 \mathrm{~ms}$ vs. unambiguous: $2211 \mathrm{~ms}$, $\left.t_{1}(19)=2.83, p_{1}=.01 ; t_{2}(13)=3.00, p_{2}=.01\right)$. They showed no significant ambiguity effect on sentence reading in the balanced ambiguous word condition (ambiguous: $2149 \mathrm{~ms}$ vs. unambiguous: $2161 \mathrm{~ms}, t \mathrm{~s}<1$ ). They were slightly faster to name words related to the context-inappropriate meanings after reading ambiguous sentences in both the biased (ambiguous: $835 \mathrm{~ms}$ vs. unambiguous: $843 \mathrm{~ms}$ ) and balanced ambiguous word condition (ambiguous: $834 \mathrm{~ms}$ vs. unambiguous: $840 \mathrm{~ms}$ ); however, the facilitation was not significant for either word type $(t s<1)$.

\subsubsection{Non-LIFG patient $M B$}

For sentence reading, MB showed an ambiguity effect of $291 \mathrm{~ms}$ in the biased ambiguous word condition (ambiguous: $2122 \mathrm{~ms}$ vs. unambiguous: $1831 \mathrm{~ms}$ ) and an ambiguity effect of $166 \mathrm{~ms}$ in the balanced ambiguous word condition (ambiguous: $1942 \mathrm{~ms}$ vs. unambiguous: $1776 \mathrm{~ms}$ ). He was slightly faster to name words related to the context-inappropriate, dominant meanings of biased ambiguous words (ambiguous: $800 \mathrm{~ms}$ vs. unambiguous: $807 \mathrm{~ms}$ ), and slower to name words related to the context-inappropriate alternative meanings of balanced ambiguous words (ambiguous: $904 \mathrm{~ms}$ vs. unambiguous: $849 \mathrm{~ms}$ ). As expected, none of his effects, on sentence reading or word naming, were abnormally large in untransformed or log-transformed data (for sentence reading: $t$ against controls $<1.49, p s>.09$; for word naming: $t$ against controls $<1$ ).

\subsubsection{LIFG patient EV}

For sentence reading, EV showed an ambiguity effect of $586 \mathrm{~ms}$ in the biased ambiguous word condition (ambiguous: $3084 \mathrm{~ms}$ vs. unambiguous: $2498 \mathrm{~ms}$ ) and an ambiguity effect of $217 \mathrm{~ms}$ in the balanced ambiguous word condition (ambiguous: $2857 \mathrm{~ms}$ vs. unambiguous: $2640 \mathrm{~ms}$ ). As expected, her ambiguity effect for biased ambiguous words was abnormally large compared to that of the controls in both untransformed and log-transformed data $(t(9)=3.28$ and 2.78 , respectively, $p s \leqslant .01)$ while her ambiguity effect for balanced ambiguous words was not ( $t$ s against controls $<1.4$, $p s \geqslant .10$ ). She was slower to name words related to the con- text-inappropriate dominant meanings of biased ambiguous words (ambiguous: $888 \mathrm{~ms}$ vs. unambiguous: $832 \mathrm{~ms}$ ), and faster to name words related to the context-inappropriate alternative meanings of balanced ambiguous words (ambiguous: $883 \mathrm{~ms}$ vs. unambiguous: $904 \mathrm{~ms}$ ); however, neither of the differences were abnormally large in either untransformed or log-transformed data $(-1<t \mathrm{~s}$ against controls $<1.2$ ).

\subsubsection{LIFG patient $M L$}

On sentence reading, ML showed an ambiguity effect of $1655 \mathrm{~ms}$ in the biased ambiguous word condition (ambiguous: $7633 \mathrm{~ms}$ for vs. unambiguous: $5978 \mathrm{~ms}$ ) and a small reverse ambiguity effect of $-29 \mathrm{~ms}$ in the balanced ambiguous word condition (ambiguous: $7028 \mathrm{~ms}$ vs. unambiguous: $7057 \mathrm{~ms}$ ). As expected, his ambiguity effect for biased ambiguous words was abnormally large compared to that of the controls in both transformed and logtransformed data $(t(9)=4.78$ and 1.90 , respectively, $p s \leqslant .05)$ while his ambiguity effect for balanced ambiguous words was not ( $t$ s against controls $<1$ ). He was $101 \mathrm{~ms}$ faster to name words related to the context-inappropriate dominant meanings of biased ambiguous words (ambiguous: $1004 \mathrm{~ms}$ vs. unambiguous: $1103 \mathrm{~ms}$ ), and $91 \mathrm{~ms}$ faster to name words related to the context-inappropriate alternative meanings of balanced ambiguous words (ambiguous: 989 ms vs. unambiguous: $1080 \mathrm{~ms}$ ); however, neither of the differences were abnormally large in either untransformed or log-transformed data $(-1.7<t \mathrm{~s}<1)$.

\subsection{Discussion of Experiment 2}

Similar to healthy younger comprehenders (e.g., Duffy et al., 1988), given prior disambiguating sentence context, the older controls were able to immediately resolve toward an alternative meaning of balanced ambiguous words, and it took them some time (mean time $=148 \mathrm{~ms}$ ) to resolve toward a subordinate meaning of biased ambiguous words. Consistent with prior aging findings (e.g., Hopkins et al., 1995; Paul, 1996), there was no significant facilitatory effect of ambiguity on word naming in the biased or balanced ambiguous word condition, suggesting that the older controls were able to successfully resolve the lexical ambiguities involving both the word types.

The patient results help to further elucidate the nature of the lexical ambiguity resolution cost associated with processing biased ambiguous words presented in prior subordinate-biasing context. Patient MB, who had relatively normal attentional control, took the same amount of time as the normal controls in resolving lexical ambiguity, both when the ambiguity involved balanced ambiguous words and when it involved biased ambiguous words. Patients EV and ML, who had impaired attentional control, took significantly more time than the normal controls in resolving lexical ambiguity only when the ambiguity involved biased ambiguous words; when the ambiguity involved balanced ambiguous words, they were as fast as the normal controls in resolving the ambiguity. As the biased ambiguous word condition induced a high level of interference from context-inappropriate dominant meanings and the bal- 
Table 3

Mean ambiguity effects (and standard deviations) for appropriate normal controls and patients on each dependent measure of the lexical ambiguity task in Experiment 2.

\begin{tabular}{llcccc}
\hline & Normal controls & Non-LIFG patient MB & LIFG patient EV & Normal controls & LIFG patient ML \\
\hline Age & 58.5 & 62 & 53 & 68.6 & 68 \\
Sentence reading & & & & \\
Biased ambiguous words & $77(148)$ & 291 & $586^{\mathrm{a}}$ & $219(287)$ & $1655^{\mathrm{a}}$ \\
Balanced ambiguous words & $29(130)$ & 166 & 217 & $-53(172)$ & -29 \\
Word naming & & & 55 & $10(67)$ & -101 \\
Biased ambiguous words & $-24(63)$ & -6 & -21 & $-10(46)$ & -91 \\
Balanced ambiguous words & $-2(92)$ & 55 & 55 & \\
\hline
\end{tabular}

a Abnormally large ambiguity effects on both untransformed and log-transformed RTs (both ps against controls <.05).

anced ambiguous word condition induced a minimal amount of interference, taken together, the results strongly argue for a role of LIFG-based attentional control processes in resolving interference from context-inappropriate meanings during the processing of lexical ambiguity in sentence context.

Interestingly, the LIFG patients showed ambiguity effects on word naming that were similar to those of the controls in both the biased and balanced ambiguous word condition. In other words, the context-inappropriate meanings were not retained at an abnormal level following ambiguity resolution in the LIFG patients. These results are not consistent with those obtained from the word-context studies, which have shown a difference between attentional control patients and normal controls in the activation of context-inappropriate meanings (Balota \& Duchek, 1991; Bedny et al., 2007). Our study differs from those studies in that we used sentences as context, with the ambiguous word being a part of the sentence so that failure to select against the context-inappropriate meaning of the word rendered the sentence nonsensical. In contrast, in the word-context studies, context words and the target word were presented in technically separate trials, making meaning selection less critical for task performance. Perhaps our strongly biasing sentence contexts and the inherently stronger trigger for meaning selection were instrumental in supporting successful lexical ambiguity resolution in our study. ${ }^{4}$

\section{General discussion}

As normal language comprehension often proceeds rapidly and effortlessly, component processes underlying comprehension must be largely automatic. But automatic comprehension does not produce an interference-free WM environment. Unintended word meanings that are strongly associated with an ambiguous word form may be highly activated in WM despite their irrelevance. To the extent that they interfere with ongoing processing, we asked what type of mechanisms is responsible for resolving the interference. Our results suggest that attentional control plays a critical role in that resolution process.

Prior research has shown that Broca's aphasic patients take an abnormally long time to select an alternative word meaning based on sentence context (Swaab et al., 1998). The deficit has been thought to arise from a delay in the process of integrating word meanings into sentence context (e.g., Hagoort, 1997; Swaab

\footnotetext{
${ }^{4}$ Novick, Kan, Trueswell, and Thompson-Schill (2009) recently reported failure of an LIFG patient IG in using referential contexts in the reanalysis of syntactic garden path sentences (e.g., Put the frog on the napkin in the box, cf. Put the frog that's on the napkin in the box). Our results, on the other hand, indicate successful use of semantic sentence contexts in the resolution of lexical ambiguities. One critical difference that might account for the discrepant findings is that our prior semantic sentence contexts were strongly biasing toward one of the alternative meanings and rendered unintended alternatives highly implausible whereas Novick et al.'s referential contexts, though providing support for the correct alternative, did not help to rule out incorrect ones.
}

et al., 1998). Although damage to Broca's area (i.e., LIFG area) is not necessarily associated with Broca's aphasia (e.g., Dronkers, Wilkins, Van Valin, Redfern, \& Jaeger, 2004), one may ask whether the exaggerated ambiguity effect that our LIFG patients showed in the high interference condition could have arisen from such a delay. If it were true that our LIFG patients were delayed in the process of integrating word meanings into sentence context, they should have shown exaggerated ambiguity effects in both the biased and balanced ambiguous word condition. The patients' normal performance in the balanced ambiguous word condition argues against this alternative explanation of our results.

An important issue is why attentional control should be necessary in the resolution of certain lexical ambiguities. That is, given that the linguistic context determines when a less preferred meaning should be selected, we might question why contextual processing within the language system would be insufficient to override a preferred meaning. Empirical data suggest that there is a limit to which context can affect word processing. When a meaning is very strongly associated with an ambiguous word form, even the strongest biasing context may fail to forestall the processing of that meaning (e.g., Kambe, Rayner, \& Duffy, 2001; Rayner, Pacht, \& Duffy, 1994; Wiley \& Rayner, 2000; see also Binder and Rayner (1999), Kellas and Vu (1999), Rayner, Binder, and Duffy (1999), Vu and Kellas (1999) for discussions). Assume a simple lexical ambiguity resolution model in which different sources of evidence are weighted equally (e.g., Duffy et al., 2001). With context pointing to one meaning but the word form strongly suggesting another, without another modulating factor, the equal weighting of the two sources of information makes it difficult, if not theoretically impossible, for the conflict to be resolved in favor of one factor vs. the other. Even in a model that allows direct suppression from contextual representations to incompatible word meaning representations (e.g., Gernsbacher \& St. John, 2001), a very strong association between the ambiguous word form and the dominant meaning could effectively eliminate the influence of context on the resolution of the lexical ambiguity (see Gernsbacher and St. John (2001) for more detail). In naturalistic settings, the ambiguous word that happens to be used may not have the strongest bias ratio and the context in which the word appears may not contain the strongest biasing information. As long as the irrelevant meaning and context are mutually conflicting and the irrelevant meaning is activated at a strong enough level in WM so as to interfere with ongoing processing, attentional control should be a critical factor in facilitating context-based ambiguity resolution.

In the current study, we focused on the processing cost associated with comprehending biased ambiguous words when prior disambiguating information is available in the sentence context and found evidence for an attentional control component to the cost. Processing costs for lexical ambiguity resolution have also been found in sentence contexts that are neutral between alternative meanings. For example, reading balanced ambiguous words presented in prior neutral context has been found to be costly 
(e.g., Duffy et al., 1988). Is implementation of attentional control part of that cost? Balanced ambiguous words appearing in a neutral context presents an interesting case of lexical ambiguity resolution, because the comparable level of association between alternative meanings and the ambiguous word form could give rise to competition for selection between the alternative meanings while the neutral context should fail to create conflict between context and alternative meanings. If conflict between context and an interfering meaning, rather than competition between alternative meanings per se, is necessary for attentional control to be triggered, the processing cost for this case of lexical ambiguity should contain no attentional control component and attentional control patients should perform at a normal level in those cases. Future studies are needed to shed light on this issue.

\section{Conclusion}

The current study provides strong evidence that LIFG-based attention control is involved in the resolution of word meaning interference during sentence comprehension. These results are consistent with accounts that emphasize the role of attentional control in lexical ambiguity resolution in particular (e.g., Shivde \& Anderson, 2001) and language processing in general (e.g., Thompson-Schill, 2005). The findings highlight the utility of a comparison of patient single cases in psycholinguistic investigation.

\section{Acknowledgments}

This research was supported by NIH Grant No. DC-00128 to Rice University. We thank Corinne Allen, Catherine Johnston, Elaine Mahoney, and Aurora Ramos for assistance with participant testing, and A. Cris Hamilton for assistance with lesion figures and sharing of test materials. We sincerely thank Keith Rayner and two anonymous reviewers for their invaluable feedback on an earlier version of this manuscript. Loan Vuong is now at the Max Planck Institute for Psycholinguistics, Nijmegen, The Netherlands.

\section{Appendix A. Supplementary material}

Supplementary data associated with this article can be found, in the online version, at doi:10.1016/j.bandl.2010.09.012.

\section{References}

Balota, D. A., Cortese, M. J. \& Wenke, D. (2001). Ambiguity resolution as a function of reading skill, age, dementia, and schizophrenia: The role of attentional control. In D. S. Gorfein (Ed.), On the consequences of meaning selection: Perspectives on resolving lexical ambiguity (pp. 87-102). Washington, DC, US: American Psychological Association.

Balota, D. A., \& Duchek, J. M. (1991). Semantic priming effects, lexical repetition effects, and contextual disambiguation effects in healthy aged individuals and individuals with senile dementia of the Alzheimer type. Brain and Language, 40, 181-201.

Balota, D. A., \& Faust, M. (2001). Attention in dementia of the Alzheimer's type. In F. Boller \& S. Cappa (Eds.) (2nd ed.. Handbook of neuropsychology: Aging and dementia (Vol. 6, pp. 51-80). Elsevier Health Sciences.

Barde, L. H. F., Schwartz, M. F., Chrysikou, E. G., \& Thompson-Schill, S. L. (2010). Reduced short-term memory span in aphasia and susceptibility to interference: Contribution of material-specific maintenance deficits. Neuropsychologia, 48, 909-920.

Barde, L. H. F., Schwartz, M. F., \& Thompson-Schill, S. (2006). The role of left inferior frontal gyrus (LIFG) in semantic short-term memory: A comparison of two case studies. Brain and Language, 99(1-2), 71-72.

Bedny, M., Hulbert, J. C., \& Thompson-Schill, S. L. (2007). Understanding words in context: The role of Broca's area in word comprehension. Brain Research, 1146, 101-114.

Binder, K. S., \& Rayner, K. (1999). Does contextual strength modulate the subordinate bias effect? A reply to Kellas and Vu. Psychonomic Bulletin E Review, 6(3), 518-522.

Burke, D. M. (1997). Language, aging, and inhibitory deficits: Evaluation of a theory. Journals of Gerontology: Series B: Psychological Sciences and Social Sciences, 52(6), P254-P264.
Crawford, J. R., Garthwaite, P. H., Howell, D. C., \& Gray, C. D. (2004). Inferential methods for comparing a single case with a control sample: Modified $t$-tests versus Mycroft et al.'s (2002) modified ANOVA. Cognitive Neuropsychology, 21, $750-755$.

Crawford, J. R., \& Howell, D. C. (1998). Comparing an individual's test score against norms derived from small samples. The Clinical Neuropsychologist (Neuropsychology Development and Cognition Section D), 12(4), 482486.

Dagerman, K. S., MacDonald, M. C., \& Harm, M. W. (2006). Aging and the use of context in ambiguity resolution: Complex changes from simple slowing. Cognitive Science, 30(2), 311-345.

Dronkers, N. F., Wilkins, D. P., Van Valin, R. D., Redfern, B. B., \& Jaeger, J. J. (2004). Lesion analysis of the brain areas involved in language comprehension. Cognition, 92, 145-177.

Duffy, S. A., Kambe, G., \& Rayner, K. (2001). The effect of prior disambiguating context on the comprehension of ambiguous words: Evidence from eye movements. In D. S. Gorfein (Ed.), On the consequences of meaning selection: Perspectives on resolving lexical ambiguity (pp. 27-43). Washington, DC: American Psychological Association.

Duffy, S. A., Morris, R. K., \& Rayner, K. (1988). Lexical ambiguity and fixation times in reading. Journal of Memory and Language, 27(4), 429-446.

Dunn, L. M., \& Dunn, L. M. (1981). Peabody picture vocabulary test - Revised. Circle Pines, MN: American Guidance Service.

Freedman, M. L., \& Martin, R. C. (2001). Dissociable components of short-term memory and their relation to long-term learning. Cognitive Neuropsychology, 18(3), 193-226.

Gernsbacher, M. A., \& St. John, M. F. (2001). Modeling suppression in lexical access. In D. S. Gorfein (Ed.), On the consequences of meaning selection: Perspectives on resolving lexical ambiguity (pp. 47-65). Washington, DC, US: American Psychological Association.

Hagoort, P. (1997). Semantic priming in Broca's aphasics at a short SOA: No support for an automatic access deficit. Brain and Language, 56(2), 287-300.

Hamilton, A. C., \& Martin, R. C. (2005). Dissociations among tasks involving inhibition: A single-case study. Cognitive, Affective \& Behavioral Neuroscience, $5(1), 1-13$

Hamilton, A. C., \& Martin, R. C. (2007). Proactive interference in a semantic shortterm memory deficit: Role of semantic and phonological relatedness. Cortex, 43(1), 112-123.

Hopkins, K. A., Kellas, G., \& Paul, S. T. (1995). Scope of word meaning activation during sentence processing by young and older adults. Experimental Aging Research, 21(2), 123-142.

Huettig, F., \& Altmann, G. T. M. (2005). Word meaning and the control of eye fixation: Semantic competitor effects and the visual world paradigm. Cognition, 96(1), B23-32.

Jonides, J., \& Nee, D. E. (2006). Brain mechanisms of proactive interference in working memory. Neuroscience, 139(1), 181-193.

Jonides, J., Smith, E. E., Marshuetz, C., Koeppe, R. A., \& Reuter-Lorenz, P. A. (1998). Inhibition in verbal working memory revealed by brain activation. Proceedings of the National Academy of Sciences, 95, 8410-8413.

Kambe, G., Rayner, K., \& Duffy, S. A. (2001). Global context effects on processing lexically ambiguous words: Evidence from eye fixations. Memory and Cognition, 29(2), 363-372.

Kellas, G., \& Vu, H. (1999). Strength of context does modulate the subordinate bias effect: A reply to Binder and Rayner. Psychonomic Bulletin E Review, 6(3), 511-517.

MacLeod, C. M. (1991). Half a century of research on the Stroop effect: An integrative review. Psychological Bulletin, 109, 163-203.

Martin, R. C., \& Lesch, M. F. (1996). Associations and dissociations between language impairment and list recall: Implications for models of STM. Models of Short-Term Memory, 149, 178.

Martin, R. C., Lesch, M. F., \& Bartha, M. C. (1999). Independence of input and output phonology in word processing and short-term memory. Journal of Memory and Language, 41(1), 3-29.

Meyer, A., Wheeldon, L., \& Krott, A. (2007). Automaticity and control in language processing. Hove and New York: Psychology Press.

Milham, M. P., Banich, M. T., \& Barad, V. (2003). Competition for priority in processing increases prefrontal cortex's involvement in top-down control: An event-related fMRI study of the Stroop task. Cognitive Brain Research, 17, 212-222.

Novick, J. M., Kan, I. P., Trueswell, J. C., \& Thompson-Schill, S. L. (2009). A case for conflict across multiple domains: Memory and language impairments following damage to ventrolateral prefrontal cortex. Cognitive Neuropsychology, 26(6), 527-567.

Novick, J. M., Trueswell, J. C., \& Thompson-Schill, S. L. (2005). Cognitive control and parsing: Reexamining the role of Broca's area in sentence comprehension. Cognitive, Affective \& Behavioral Neuroscience, 5(3), 263-281.

Paul, S. T. (1996). Search for semantic inhibition failure during sentence comprehension by younger and older adults. Psychology and Aging, 11, $10-20$.

Rayner, K., Binder, K. S., \& Duffy, S. A. (1999). Contextual strength and the subordinate bias effect: Comment on Martin, Vu, Kellas, and Metcalf. The Quarterly Journal of Experimental Psychology A: Human Experimental Psychology, 52(4), 841-852.

Rayner, K., Pacht, J. M., \& Duffy, S. A. (1994). Effects of prior encounter and global discourse bias on the processing of lexically ambiguous words: Evidence from eye fixations. Journal of Memory and Language, 33, 527-544. 
Roach, A., Schwartz, M. F., Martin, N., Grewal, R. S., \& Brecher, A. (1996). The Philadelphia naming test: Scoring and rationale. Clinical Aphasiology, 24, 121-134.

Rosinski, R. R., Golinkoff, R. M., \& Kukish, K. S. (1975). Automatic semantic processing in a picture-word interference task. Child Development, 46, 247-253.

Shivde, G., \& Anderson, M. C. (2001). The role of inhibition in meaning selection: Insights from retrieval-induced forgetting. In D. S. Gorfein (Ed.), On the consequences of meaning selection: Perspectives on resolving lexical ambiguity (pp. 175-190). Washington, DC, US: American Psychological Association.

Simpson, G. B., \& Adamopoulos, A. C. (2001). Repeated homographs in word and sentence contexts: Multiple processing of multiple meanings. In D. S. Gorfein (Ed.), On the consequences of meaning selection: Perspectives on resolving lexical ambiguity (pp. 105-117). Washington, DC, US: American Psychological Association.

Simpson, G. B., \& Kang, H. (1994). Inhibitory processes in the recognition of homograph meanings. In D. Dagenbach \& T. H. Carr (Eds.), Inhibitory processes in attention, memory, and language (pp. 359-381). San Diego: Academic Press.

Stroop, J. R. (1935). Studies of interference in serial verbal reactions. Journal of Experimental Psychology: General, 18, 643-662.

Swaab, T. Y., Brown, C., \& Hagoort, P. (1998). Understanding ambiguous words in sentence contexts: Electrophysiological evidence for delayed contextual selection in Broca's aphasia. Neuropsychologia, 36(8), 737-761.
Swaab, T. Y., Brown, C., \& Hagoort, P. (2003). Understanding words in sentence contexts: The time course of ambiguity resolution. Brain and Language, 86(2), 326-343.

Swinney, D. A. (1979). Lexical access during sentence comprehension: (Re) consideration of context effects. Journal of Verbal Learning and Verbal Behavior 18(6), 645-659.

Thompson-Schill, S. L. (2005). Dissecting the language organ: A new look at the role of Broca's area in language processing. In A. Cutler (Ed.), Twenty-first century psycholinguistics: Four cornerstones (pp. 173-189). Hillsdale, NJ: Lawrence Erlbaum Associates.

Thompson-Schill, S. L., Jonides, J., Marshuetz, C., Smith, E. E., D’Esposito, M., Kan, I. P., et al. (2002). Effects of frontal lobe damage on interference effects in working memory. Cognitive, Affective E Behavioral Neuroscience, 2(2), 109-120.

Verhaeghen, P., \& De Meersman, L. (1998). Aging and the Stroop effect: A metaanalysis. Psychology and Aging, 13, 120-126.

Vu, H., \& Kellas, G. (1999). Contextual strength modulates the subordinate bias effect: Reply to Rayner, Binder, and Duffy. The Quarterly Journal of Experimenta Psychology A, 52(4), 853-855.

Wiley, J., \& Rayner, K. (2000). Effects of titles on the processing of text and lexically ambiguous words: Evidence from eye movements. Memory and Cognition, 28(6) 1011-1021. 